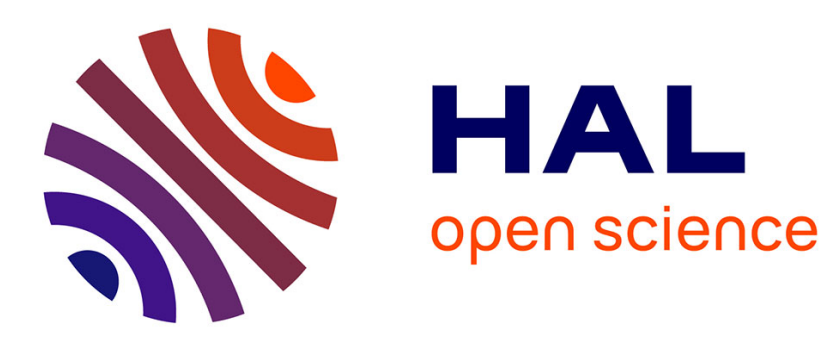

\title{
Inherent relevance of MRMT models to concentration variance and mixing-induced reactivity
}

\author{
Tristan Babey, Jean-Raynald de Dreuzy, Alain Rapaport, Alejandro \\ Rojas-Palma
}

\section{- To cite this version:}

Tristan Babey, Jean-Raynald de Dreuzy, Alain Rapaport, Alejandro Rojas-Palma. Inherent relevance of MRMT models to concentration variance and mixing-induced reactivity. Advances in Water Resources, 2017, 110, pp.291-298. 10.1016/j.advwatres.2017.09.024 . insu-01609279

\section{HAL Id: insu-01609279 \\ https://hal-insu.archives-ouvertes.fr/insu-01609279}

Submitted on 3 Oct 2017

HAL is a multi-disciplinary open access archive for the deposit and dissemination of scientific research documents, whether they are published or not. The documents may come from teaching and research institutions in France or abroad, or from public or private research centers.
L'archive ouverte pluridisciplinaire $\mathbf{H A L}$, est destinée au dépôt et à la diffusion de documents scientifiques de niveau recherche, publiés ou non, émanant des établissements d'enseignement et de recherche français ou étrangers, des laboratoires publics ou privés. 


\section{Accepted Manuscript}

Inherent relevance of MRMT models to concentration variance and mixing-induced reactivity

Tristan Babey, Jean-Raynald de Dreuzy, Alain Rapaport, Alejandro Rojas-Palma

PII:

DOI:

Reference:

To appear in:

Received date:

Revised date:

Accepted date:
S0309-1708(17)30366-4

10.1016/j.advwatres.2017.09.024

ADWR 2958

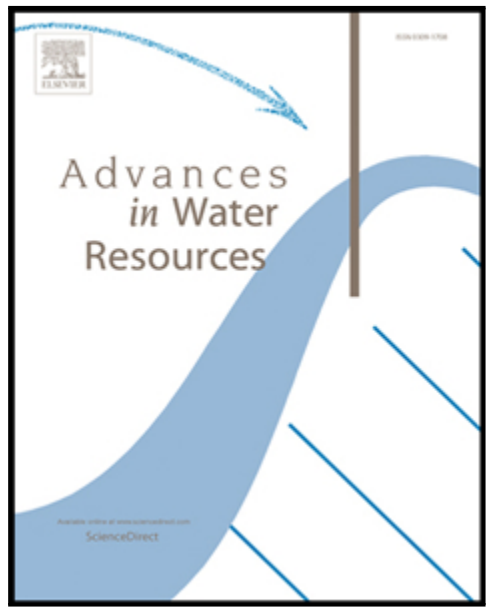

Please cite this article as: Tristan Babey, Jean-Raynald de Dreuzy, Alain Rapaport, Alejandro Rojas-Palma, Inherent relevance of MRMT models to concentration variance and mixinginduced reactivity, Advances in Water Resources (2017), doi: 10.1016/j.advwatres.2017.09.024

This is a PDF file of an unedited manuscript that has been accepted for publication. As a service to our customers we are providing this early version of the manuscript. The manuscript will undergo copyediting, typesetting, and review of the resulting proof before it is published in its final form. Please note that during the production process errors may be discovered which could affect the content, and all legal disclaimers that apply to the journal pertain. 


\section{Highlights}

- Generic models of concentration variability in diffusion-dominated porosities.

- Mobile/immobile models like MRMT are relevant for concentration mean and variance.

- Combinations of porosities in MRMT inherently conserve concentration variance.

- Equivalent MRMT models approximate well homogeneous and heterogeneous reactivities.

- Equivalent MRMT should be of the same minimal dimension as the input/output system.

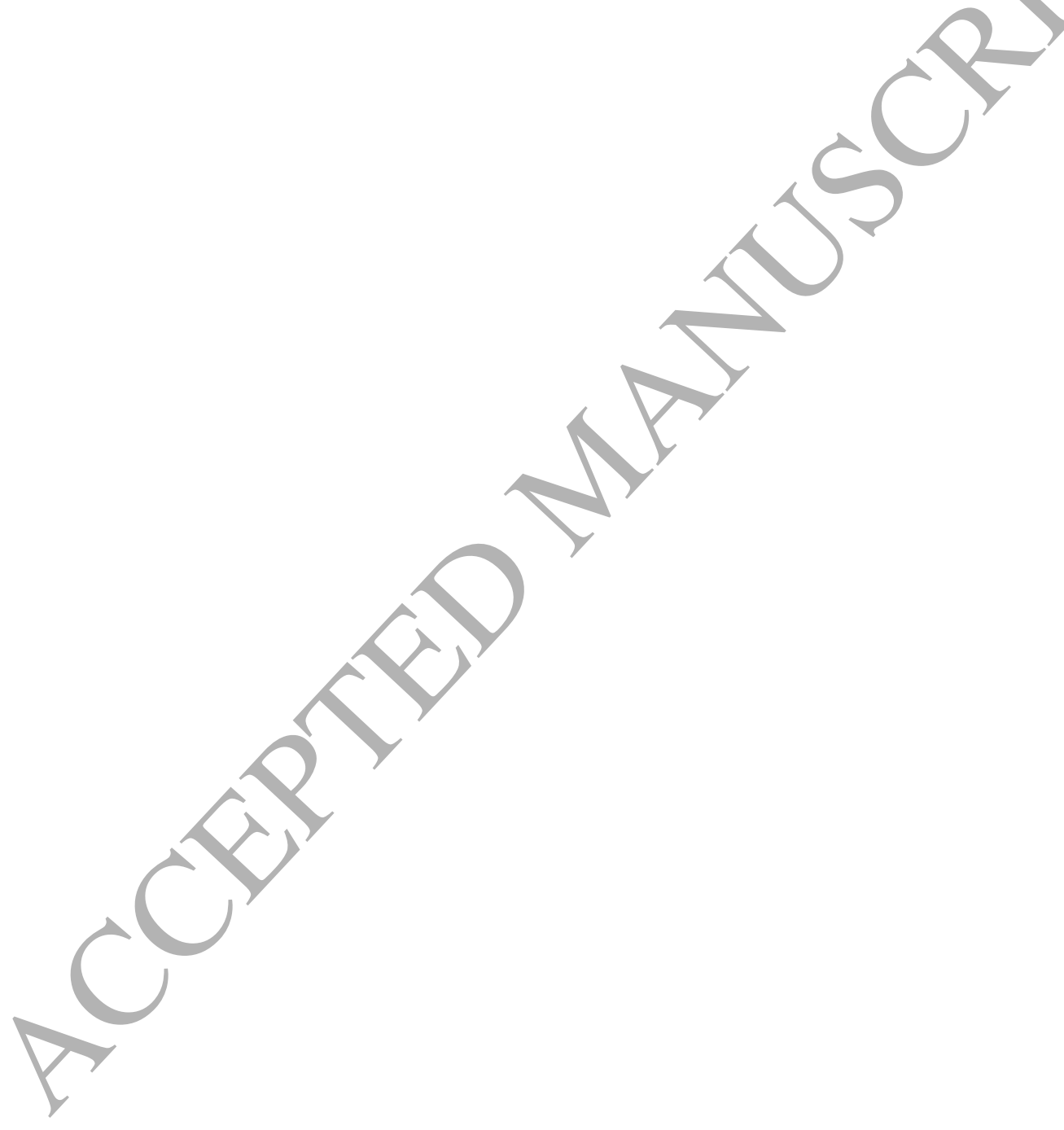




\section{Inherent relevance of MRMT models to concentration variance and mixing-induced reactivity}

\footnotetext{
Tristan Babey ${ }^{\mathrm{a}}$, Jean-Raynald de Dreuzy ${ }^{\mathrm{a}}$, Alain Rapaport ${ }^{\mathrm{b}}$, Alejandro Rojas-Palma ${ }^{\mathrm{c}}$

${ }^{a}$ Géosciences Rennes UMR CNRS 6118, Campus de Beaulieu, Université de Rennes 1, 35042 Rennes cedex, France

${ }^{b}$ UMR 729 INRA/SupAgro MISTEA (Mathématiques, Informatique et STatistique pour l'Environnement et l'Agronomie), 2 pl. Viala, 34060 Montpellier, France

${ }^{c}$ Departamento de Matemática, Física y Estadística, Universidad Católica del Maule, Talca, Chile
}

\section{Abstract}

Several anomalous transport approaches have been developed to model the interaction between fast advectively-dominated transport in well-connected porosity and fracture structures and slow diffusively-dominated transport in poorly-connected or low-permeability ones. Among them, the Multi-Rate Mass Transfer approach (MRMT) represents the anomalous dispersion along the main flow paths (mobile zone) induced by a large distribution of first-order exchanges with immobile zones. Even though MRMTs have been developed for conservative transport processes in the mobile zone, we demonstrate that they also conserve the variance of the concentration distribution in the immobile zones, and, hence, pertain to mixing induced reactivity. This property is established whatever the organization of the immobile zones and whatever the injection and sampling conditions in the mobile zone. It inherently derives from the symmetry properties of the diffusion operator in the immobile zones, but cannot be directly extended to heterogeneous dispersive processes in the mobile zone.

Keywords: Anomalous transport, Reactive transport, Multi-Rate Mass Transfer models, Heterogeneous geological media

\section{Highlights}

1. Generic models of concentration variability in diffusion-dominated porosities.

2. Mobile/immobile models like MRMT are relevant for concentration mean and variance.

3. Combinations of porosities in MRMT inherently conserve concentration variance.

4. Equivalent MRMT models approximate well homogeneous and heterogeneous reactivities.

5. Equivalent MRMT should be of the same minimal dimension as the input/output system.

Email address: jean-raynald.de-dreuzy@univ-rennes1.fr (Jean-Raynald de Dreuzy) 


\section{Introduction}

Dispersion in geological media derives from simple advective and diffusive processes in complex porous and fracture structures [Gelhar and Axness, 1983]. Solutes are delayed by trapping in poorly connected porosity structures and dispersed by exchanges with fast transport in localized channels. Such structures are found as intragranular clay particles [Scheibe et al., 2013], stagnant zones in carbonates [Bijeljic et al., 2013], poorly connected fracture clusters [Davy et al., 2010], hydraulic dead ends in fractures [Park et al., 2003] or incomplete dissolution patterns [Luquot et al., 2014]. Diffusion and trapping are significant and may induce non-Fickian anomalously slow and highly-dispersed transport [Bouchaud and Georges, 1990; Dentz and Berkowitz, 2003; Havlin and Ben-Avraham, 1987]. Anomalous transport has been reported both in porous and in fractured media at multiple scales from laboratory experiments [Soler-Sagarra et al., 2016; Zinn et al., 2004; Knorr et al., 2016], advanced analysis of microtromography images [Gjetvaj et al., 2015; Gouze et al., 2008], field experiments [Greskowiak et al., 2011; Le Borgne and Gouze, 2008] and numerical simulations [de Dreuzy and Carrera, 2016; Fernandez-Garcia et al., 2009; Lichtner and Kang, 2007; Roubinet et al., 2013; Willmann et al., 2010].

Several conceptual frameworks have been developed to model anomalous transport [Benson et al., 2000; Berkowitz et al., 2006; de Dreuzy and Carrera, 2016; Neuman and Tartakovsky, 2009]. Among them, the mobile-immobile Multi-Rate Mass Transfer models (MRMT) [Carrera et al., 1998; Haggerty and Gorelick, 1995 does not only propose efficient characterization and upscaling methodologies [Willmann et al., 2008; Babey et al., 2015; Rapaport et al., 2017] but also a natural bridge to equivalent concentration distributions, which relevance can be assessed to model reactive transport [Donado et al., 2009; Henri and Fernandez-Garcia, 2015; Sanchez-Vila et al., 2010; Soler-Sagarra et al., 2016]. Synthetic experiments have shown that MRMT models provide close approximations of bulk reactivity even in non-linear equilibrium and kinetically-controlled cases [Babey et al., 2016]. This has been linked to the empirical observation that MRMT models do not only conserve mass by construction but also the porosity weighted integral of concentrations squared [de Dreuzy et al., 2013], which is directly linked to mixing-induced reactivity through the scalar dissipation rate [Le Borgne et al., 2010].

While the conservation of the porosity weighted integral of concentrations squared has so far been reported from numerical experiments in a couple of specific cases, we provide here the full demon- 
stration of its conservation. This property is inherent to the formalism of the mobile/immobile models (including MRMT) providing the model to be minimal. It derives from the conservation of mass in the mobile zone and from the expression of the immobile concentrations as the direct difference between immobile and mobile concentrations. It does not require any additional condition. The demonstration applies to any type of diffusively-dominated porosity structure exchanging with advectively-dominated transport identified with the so-called mobile zones. We shortly discuss the implications on the general relevance of MRMT models to chemical transport.

\section{Dynamics of concentrations in mobile/immobile models}

In this section, we recall the general framework proposed by Babey et al. [2015] to model solute transport resulting from the interactions between a mobile zone and a finite number of $n$ immobile zones. This framework identified as the Structured INteracting Continua (SINC) model was introduced as an extension of the classic Multiple INteracting Continua (MINC) model [Pruess and Narasimhan, 1985; Karimi-Fard et al., 2006]. Transport is dominated by advection along the "mobile zone", and solutes are exchanged by diffusion with and between the different "immobile zones". Immobile zones can display any connectivity patterns coming, for example, from the discretization of diffusion within dead-ends of fractures or pore clusters [Davy et al., 2010; Gouze et al., 2008; Karimi-Fard and Durlofsky, 2016], alluvial architectures [Zhang et al., 2013, 2014], heterogeneous porous media [Li et al., 2011; Tyukhova et al., 2015; Tyukhova and Willmann, 2016] or multi-porosity reservoirs [Geiger et al., 2013]. The SINC model is formalized as:

$$
\frac{\partial C}{\partial t}+\Phi^{-1} M C=B L\left(c_{1}\right)
$$

5 where $C$ is the column vector made up of the solute concentrations in the mobile and immobile zones:

$$
C=\left[\begin{array}{ccc}
c_{1}(r, t) & \ldots & c_{n+1}(r, t)
\end{array}\right]^{T} .
$$

$c_{1}(r, t)$ is the concentration in the mobile zone and $c_{i}(r, t)$ with $i=2 \ldots n+1$ are the concentrations in the $n$ immobile zones. $L$ is the advective-dispersive transport operator in the mobile zone:

$$
L\left(c_{1}\right)=-\frac{1}{\phi_{1}} \nabla \cdot\left(q c_{1}\right)+\nabla \cdot\left(D_{m} \nabla c_{1}\right)
$$


where $\phi_{1}, q$ and $D_{m}$ are the uniform porosity, Darcian flow and diffusion-dispersion tensor in the mobile zone. $B$ is the restriction vector to the mobile zone:

$$
B=\left[\begin{array}{llll}
1 & 0 & \ldots & 0
\end{array}\right]^{T} .
$$

$\Phi$ is the porosity matrix of size $(n+1, n+1)$ which diagonal coefficients are the porosities of the different zones $\phi_{i}$ :

$$
\Phi=\operatorname{diag}\left(\left[\begin{array}{lll}
\phi_{1} & \ldots & \phi_{n+1}
\end{array}\right]\right) .
$$

Finally, the matrix $M$ of size $(n+1, n+1)$ is the operator describing the diffusive-like exchanges between the different zones. $M$ can be compared to a weighted adjacency matrix [Godsil and Royle, 2001] as its coefficients correspond to rates of mass exchanges. Because it expresses a diffusion process, $M$ is a symmetric M-matrix which rows sum to zero. Eq. 1 can be rewritten to highlight the interactions between the concentrations of the different zones:

$$
\frac{\partial C}{\partial t}-A C=B L\left(c_{1}\right)
$$

where $A$ is the interaction matrix that synthesizes porosity and diffusive mass exchange effects:

$$
A=-\Phi^{-1} M
$$

Except in the specific case of uniform porosity, $A$ is not symmetric.

MRMT models with a finite number of immobile zones can be expressed within the SINC framework as the subset of models with only mobile-immobile connections, with the corresponding porosity and mass exchange matrices $\Phi$ and $M$ given by:

$$
\begin{aligned}
& \Phi=\operatorname{diag}\left(\left[\begin{array}{lll}
\phi_{1} & \ldots & \phi_{n+1}
\end{array}\right]\right) \\
& M(i, j)=0 \text { for } i>1, j>1 \text { and } i \neq j \\
& M(i, 1)=M(1, i)=-\phi_{i} \alpha_{i-1} \text { for } i>1 \\
& M(i, i)=-\sum_{j, j \neq i} M(i, j)
\end{aligned}
$$

where $\alpha_{i}$ and $\phi_{i}$ are the rates of exchanges and porosities of the MRMT model Haggerty and Gorelick [1995]. 


\section{Conservation of concentration variance by MRMT models}

Babey et al. [2015] and Rapaport et al. [2017] have demonstrated that any mobile-immobile model identified as SINC in the previous section, i.e. whatever the connectivity of its immobile zones, is equivalent to a unique (up to the numbering of the immobile zones) MRMT model, proving the original model to be minimal. Equivalence between SINC and MRMT is meant here as the same number of immobile zones and the same partition of concentrations between the mobile and immobile zones:

$$
\begin{aligned}
c_{1}(r, t) & =\bar{c}_{1}(r, t) \\
\sum_{i=2}^{n+1} \phi_{i} c_{i}(r, t) & =\sum_{i=2}^{\bar{n}+1} \bar{\phi}_{i} \bar{c}_{i}(r, t)
\end{aligned}
$$

where $c_{i}, \phi_{i}$ and $n$ are respectively the concentrations, porosities and total number of immobile zones for SINC, and $\bar{c}_{i}, \bar{\phi}_{i}$ and $\bar{n}$ are their counterparts for the equivalent MRMT model.

When the original SINC model is not minimal, the variance of concentration is not conserved as shown by the counterexample of Appendix A1. The absence of minimality cannot be detected with any straightforward criterion as it concerns the exchanges of the overall immobile structure with the mobile zone. The example of Appendix A1 does not show any visual symmetry or redundant structure although it is not minimal. Minimality of the system can however be tested by its controllability and observability properties [Andréa-Novel and de Lara, 2013] (here observability is equivalent to controllability because input and output are in the same mobile zone: see [Rapaport et al., 2017]). A system is said controllable if, for all couple of state vectors $\left(C^{a}, C^{b}\right)$, there exists a finite time $T \geq 0$ and a input concentration $c_{1}^{\text {in }}(\cdot)$ defined on $[0, T]$ such that, applying this input function, the solution $C(\cdot)$ with initial condition $C(0)=C^{a}$ satisfies $C(T)=C^{b}$. Controllability is checked by the algebraic condition that the controllability matrix given by

$$
\mathcal{C}=\left[B, A B, \ldots A^{n} B\right]
$$

is full rank. The lack of minimality means that there is some redundancy in the SINC structure in terms of exchange terms (see example in Appendix A1). When the original model is non minimal, it is however possible to reduce the model to an equivalent minimal one ([Chen, 1999]), which can 
be sought here as a SINC model with less immobile zones $(\bar{n}<n)$ before applying the algorithm given in [Rapaport et al., 2017] to then obtain an equivalent MRMT model with $\bar{n}$ immobile zones.

After recalling how MRMT models can be built from minimal SINC models, we show that MRMT models inherently preserve the porosity weighted integral of the concentrations squared:

$$
\sum_{i=1}^{n+1} \phi_{i} c_{i}^{2}=\sum_{i=1}^{\bar{n}+1} \bar{\phi}_{i} \bar{c}_{i}^{2}
$$

or equivalently in algebraic form:

$$
C^{T} \Phi C=\bar{C}^{T} \bar{\Phi} \bar{C}
$$

with $C$ and $\Phi$ the concentration vector and diagonal porosity matrix for SINC, and $\bar{C}$ and $\bar{\Phi}$ their counterparts for the equivalent MRMT model.

Assuming the identity of concentration partition (Eqs. 9-10), the equivalent MRMT model to a SINC model writes:

$$
\frac{\partial \bar{C}}{\partial t}-\bar{A} \bar{C}=B L\left(c_{1}\right)
$$

where $\bar{A}$ and $\bar{C}$ are the interaction matrix and concentration vector for the equivalent MRMT model:

$$
\left\{\begin{array}{l}
\bar{A}=R A R^{-1} \\
\bar{C}=R C
\end{array}\right.
$$

with $R$ the transformation matrix from SINC to MRMT. Concentrations $\bar{C}$ are qualified as semilocal as they are still concentrations from the dimension point of view but are only combinations of effective concentrations $C$. $R$ derives from the diagonalization of the sub-matrix $A_{S}=A(2$ : $n+1,2: n+1)$, which describes exclusively the exchanges between the immobile zones, into $\bar{A}_{S}=\bar{A}(2: n+1,2: n+1):$

$$
\bar{A}_{S}=R_{S} A_{S} R_{S}^{-1}
$$

where $R_{S}$ is the matrix composed of the eigenvectors of $A_{S}$. The eigenvalues of $A_{S}$ (diagonal coefficients of $\overline{A_{S}}$ ) are the rates $\alpha_{i}$ of the equivalent MRMT model (Eq. 8). To be representative 
of a MRMT model, $\bar{A}$ must have the same shape as $A$ described by Eq. 7 :

$$
\bar{A}=-\bar{\Phi}^{-1} \bar{M}
$$

where $\bar{\Phi}$ and $\bar{M}$ are the porosity and mass exchange matrices given by Eq. 8. Rapaport et al. [2017] have shown that this condition is fulfilled only if the pair $(A, B)$ is controllable. The full transformation matrix $R$ then writes:

$$
R=\left[\begin{array}{cc}
1 & 0 \\
0 & -R_{S} \bar{A}_{S}{ }^{-1} \operatorname{diag}\left(R_{S}^{-1} A(2: n+1,1)\right)
\end{array}\right] .
$$

$R$ verifies the conservation of concentrations in the mobile zone (Eq. 9). By construction, $R$ also ensures that any uniform concentration profile in SINC remains equally uniform in its equivalent MRMT:

$$
\left[\begin{array}{c}
1 \\
\vdots \\
1
\end{array}\right]=R\left[\begin{array}{c}
1 \\
\vdots \\
1
\end{array}\right] y
$$

From this formulation of the equivalence between SINC and MRMT, we derive the expressions of $\bar{\Phi}$ and $\bar{M}$ as functions of $\Phi$ and $M$ and of the transformation matrix $R$. To this end, we develop the expression of $\bar{A}$ in Eq. 15 by introducing the definition of $A$ (Eq. 7):

$$
\bar{A}=-R \Phi^{-1} M R^{-1}
$$

and introduce the matrices $T=R \Phi^{-1 / 2}$ and $S=\Phi^{-1 / 2} M \Phi^{-1 / 2}$ such that

$$
\begin{aligned}
\bar{A} & =-T S T^{-1} \\
& =-T T^{T}\left(T^{-1}\right)^{T} S T^{-1} .
\end{aligned}
$$

We note $T_{S}$ and $S_{S}$ the sub-matrices of $T$ and $S$ made up of their last $n$ rows and columns. As $\bar{A}_{S}$ is diagonal, $T_{S}$ diagonalizes the matrix $S_{S}$ :

$$
T_{S} S_{S} T_{S}^{-1}=R_{S} A_{S} R_{S}^{-1}=\bar{A}_{S}
$$

and, because $S_{S}$ is symmetric, and $\bar{A}_{S}$ has distinct eigenvalues, $T_{S}$ is of the form $D U$ where $D$ is a diagonal matrix and $U$ is a unitary matrix. $T_{S} T_{S}^{T}$ is in turn equal to a positive diagonal matrix 
$D^{2}$. Due to the structure of the matrix $R$, one has:

$$
T T^{T}=\left[\begin{array}{cc}
1 / \phi_{1} & 0 \\
0 & T_{S} T_{S}^{T}
\end{array}\right] .
$$

Because $T T^{T}$ is positive and diagonal, and because $\left(T^{-1}\right)^{T} S T^{-1}$ is symmetric, they can be identified respectively to $\bar{\Phi}^{-1}$ and $\bar{M}$ of Eq. 17 :

$$
\begin{gathered}
\bar{\Phi}=\left(T T^{T}\right)^{-1}=\left(R^{-1}\right)^{T} \Phi R^{-1} \\
\bar{M}=\left(T^{-1}\right)^{T} S T^{-1}=\left(R^{-1}\right)^{T} M R^{-1} .
\end{gathered}
$$

It should be noted that the transformations of porosities and mass exchange rates from SINC to MRMT are identical and directly derive from the change of basis of the concentrations given by Eqs. 15, 18 and 19. The conservation of the porosity weighted integral of concentrations squared of Eq. 13 derives from the consistency between the change of basis of concentrations and porosities (Eqs. 15 and 24):

$$
\begin{aligned}
\bar{C}^{T} \bar{\Phi} \bar{C} & =C^{T} R^{T}\left(R^{-1}\right)^{T} \Phi R^{-1} R C \\
& =C^{T} \Phi C .
\end{aligned}
$$

As this conservation directly results from the construction of MRMT without any additional constraint, it is an inherent property of MRMT models of finite dimension (i.e. having a finite number of immobile zones). It fundamentally derives from the symmetry of the exchange matrix $M$ of the mobile-immobile/model, a fundamental property of the diffusion operator, which conditions the orthogonality of the matrix $T$, a property essential to the demonstration. The conservation of the porosity weighted/integral of concentrations squared can be extended to all porosity structures that are equivalent to the same MRMT, i.e. that have the same mobile concentrations (Appendix A2).

The same conservation of the porosity-weighted sum of concentrations squared can also be established for the uniform radial diffusion in the immobile zone whatever its dimension (1, 2 or 3), where the equivalent MRMT derives from a separation of variables methodology [Haggerty and Gorelick, 1995; Carrera et al., 1998]. As the former demonstration requires the number of immobile zones to be finite, we provide for an alternative demonstration in Appendix A3. we 
show that this conservation derives again from the symmetry of the diffusion operator. In fact, the orthogonality of the basis function of the diffusion equation in radially uniform media removes cross products between basis functions. Proper choice of eigenvector normalization also derives from the conservation of uniform concentration profiles as in Eq. 19.

\section{Discussion and conclusions}

Eqs. 10 and 12 show that the first and second moments of the concentration distribution in the immobile zones are conserved in the passage from the porosity structure to its equivalent MRMT model. This result pertains to any organization of the immobile zones and any injection conditions. It is also valid whether the initial porosity organization is described by continuous or discrete formalisms, as long as injection and sampling are carried out exclusively in the mobile zone. This is typically the case in tracer tests where tracers are injected into and collected from the flowing/mobile zone.

As both the first and second moments of the concentration distribution in the immobile zones are conserved in MRMT, so is the concentration variance and, hence, the scalar dissipation rate that strongly conditions mixing-induced reactivity [Le Borgne et al., 2011]. In fact, the reaction rate can be expressed as the product of the scalar dissipation rate by a chemical term, which depends on the nature of the reaction [De Simoni et al., 2005, 2007; Rubin, 1983]. For advective-diffusive transport without solute flux across boundaries, the scalar dissipation rate is inversely proportional to half of the second moment of the concentration distribution [Le Borgne et al., 2010]. Larger concentration variances result in stronger mixing potential between higher and lower concentration values and promote homogeneous reactivity (reactivity in solution). By reducing concentration variances, diffusive processes induced by mobile-immobile mass exchanges thus promote reactivity and, by consequence, reduce further mixing and reaction potentials.

The scalar dissipation rate only differs from the reaction rate by a chemical term that derives from the nonlinearity of the reactivity in the reactant concentrations [De Simoni et al., 2005]. Numerical simulations have however shown that the influence of this chemical term remains limited for most immobile porosity structures and reaction types also extending to heterogeneous reactivity including sorption and precipitation/dissolution [Babey et al., 2016]. The relevance of MRMT to model reactive transport fundamentally comes from the representativity for the concentration 
distribution of its mean and variance in diffusively-dominated conditions. Although concentration distributions are more complex than Gaussian, they do not differ much and lead to close approximations. As diffusion further smoothens concentration profiles, approximations become even closer with time.

Use of Multi-Rate Mass Transfer models for reaction rate predictions benefits from the development of different numerical approaches proposed for extending classical advection dispersion schemes in the mobile zone to account for exchanges with immobile zones. Approaches have been proposed based either on Eulerian schemes [Silva et al., 2009] or Lagrangian schemes [Noétinger et al., 2016; Roubinet et al., 2013]. They may be used to assess the concentration distribution and the associated reactivity rate either in a postprocessing step when transport and reactivity can be fully decoupled [Donado et al., 2009; Willmann et al., 2010] or with classical sequential or global implicit coupling methods otherwise [de Dieuleveult et al., 2009; Steefel et al., 2005]. Concentration gradients necessary for computing local reactivity rates may be obtained directly with appropriate schemes [Beaudoin et al., 2017] or eventually derived from finite-differencing the concentration field.

While diffusive processes in poorly connected porosity structures smoothen concentration gradients, dispersive processes modeled at the fundamental scale of the spatial and temporal variabilities of the velocity field will tend, on the contrary, to retain concentration differences [Le Borgne et al., 2011; de Dreuzy et al., 2012]. When diffusive and dispersive processes occur in the same domain like in strongly heterogeneous porous media [Delhomme, 1979], mixing eventually results from the interplay between the spatial and temporal fluctuations of the velocity field at the origin of dispersion and the diffusive exchanges in the least pervious zones [de Dreuzy et al., 2012; Pool et al., 2015]. Multi-Rate Mass Transfer models inherently built for diffusive processes no longer hold to model dispersion/[de Dreuzy and Carrera, 2016] and other dynamic analysis of the concentration field based for examples on lamellas deformed by the velocity fluctuations may be used to approximate the concentration field [Le Borgne et al., 2015]. Further research is thus needed to explore the overall effect of combined dispersion and exchanges with diffusive zones.

In the restrictive case where macroscopic dispersion is dominantly induced by exchanges between advective and diffusive zones, conservative tracer testing already contains most of the information 
necessary to evaluate the physical control of reactivity. Simple breakthrough curves are sufficient to calibrate MRMT models and produce predictions of the immobile concentration distribution, and reactive transport can be approached by simply coupling MRMT models with the targeted reactive processes.

\section{Acknowledgments}

The ANR is acknowledged for its funding through its project Soil $\mu 3 \mathrm{D}$ under the no. ANR-15CE01-0006. 


\section{Appendix A1: Controllability of diffusive porosity structures}

In this example, we show how the lack of controllability and minimality precludes the conservation of the concentration variance. We consider four zones with the porosities $\phi_{1}=1, \phi_{2}=1, \phi_{3}=2$, $\phi_{4}=3$ and the exchange coefficients (coefficients of the matrix $M$ of Eq. 1) $m_{12}=1, m_{13}=2$, $m_{14}=3, m_{23}=3, m_{24}=3$. The first zone is the mobile zone, the three other ones are immobile zones. The sum of the concentrations squared weighted by the porosities is

$$
\Sigma=C^{t} \Phi C=C_{1}^{2}+C_{2}^{2}+2 C_{3}^{2}+3 C_{4}^{2} .
$$

The PDE of Eq. 6 can be written at a given position along the mobile zone as an input-output representation such as:

$$
\frac{d C}{d t}=A C+B C_{\text {in }}, \quad C_{\text {out }} \neq B^{T} \mathcal{G}
$$

where $C_{\text {in }}, C_{\text {out }}$ are respectively the input and output concentrations. Only advection is considered in the mobile zone. In the specific case considered $A$ and $B$ are given by:

$$
A=\left[\begin{array}{rrrr}
-7 & 1 & 2 & 3 \\
1 & -7 & 3 & 3 \\
1 & \frac{3}{2} & -\frac{5}{2} & 0 \\
1 & 1 & 0 & -2
\end{array}\right], \quad B=\left[\begin{array}{l}
1 \\
0 \\
0 \\
0
\end{array}\right]
$$

At first look, this structure does not exhibit any special property or symmetry that could make believe that the input-output system is non minimal. The pair $(A, B)$ is however non controllable, even though one can check that the sub-matrix $A(2: 4,2: 4)$ has distinct eigenvalues. It can be shown that:

$$
A B=\left[\begin{array}{r}
-7 \\
1 \\
1 \\
1
\end{array}\right], A^{2} B=\left[\begin{array}{r}
55 \\
-8 \\
-8 \\
-8
\end{array}\right]=-B-8 A B
$$

and that the rank of the matrix $\mathcal{C}$ defined in Eq. (11) is 2, where it should be full ranked. Therefore, the system admits a minimal representation of dimension only 2 . The equivalent immobile zone 
can be found by merging the immobile zones in one with a porosity

$$
\bar{\phi}=\phi_{2}+\phi_{3}+\phi_{4}=6
$$

and an equivalent concentration

$$
\bar{C}=\frac{\phi_{2} C_{2}+\phi_{3} C_{3}+\phi_{4} C_{4}}{\bar{\phi}}=\frac{C_{2}+2 C_{3}+3 C_{4}}{6} .
$$

One can check that $\left(C_{1}, \bar{C}\right)$ is solution of the differential system:

$$
\frac{d}{d t}\left[\begin{array}{c}
C_{1} \\
\bar{C}
\end{array}\right]=\left[\begin{array}{cc}
-7 & 6 \\
1 & -1
\end{array}\right]\left[\begin{array}{c}
C_{1} \\
\bar{C}
\end{array}\right]+\left[\begin{array}{l}
1 \\
0
\end{array}\right] C_{i n}
$$

that provides an equivalent input-output system. The sum of the concentrations squared weighted by the porosities in the equivalent representation is:

$$
\bar{\Sigma}=C_{1}^{2}+\bar{\phi} \bar{C}^{2}=C_{1}^{2}+\frac{\left(C_{2}+2 C_{3}+3 C_{4}\right)^{2}}{6}
$$

which differs from $\Sigma$, because here the original-representation is not minimal.

Indeed, the matrix $R$ given in Eq. 18 is in this example:

$$
R=\left[\begin{array}{llll}
1 & 0 & 0 & 0 \\
0 & 0 & 1 & 0 \\
0 & 0 & 1 & 0 \\
0 & 0 & 1 & 0
\end{array}\right]
$$

270 which fails to be a transformation matrix to MRMT. One has

$$
R_{S}=\left[\begin{array}{ccc}
0.8626094 & -0.4492446 & -0.4133648 \\
-0.2886751 & -0.5773503 & -0.8660254 \\
-0.0600512 & -0.6918374 & 0.7518886
\end{array}\right]
$$

that diagonalizes the sub-matrix $A_{S}$ :

$$
R_{S}\left[\begin{array}{ccc}
-7 & 3 & 3 \\
\frac{3}{2} & -\frac{5}{2} & 0 \\
1 & 0 & -2
\end{array}\right] R_{S}^{-1}=\left[\begin{array}{ccc}
-8.2603986 & 0 & 0 \\
0 & -1 & 0 \\
0 & 0 & -2.2396014
\end{array}\right]
$$


but the vector

$$
R_{S}^{-1} A(2: 4,1)=\left[\begin{array}{c}
0 \\
-1.7320508 \\
0
\end{array}\right]
$$

has null entries. In [Rapaport et al., 2017], it is proved that for matrices of the form of Eq. (7) the vector $R_{S}^{-1} A(2: n+1,1)$ has non-null entries exactly when the pair $(A, B)$ is controllable.

One may argue that having the pair $(A, B)$ non controllable is a very particular and rare case (as the property of having a singular controllability matrix of Eq. (11) is non generic in the set of matrices $A$ of the form of Eq. (7)), but the distance to uncontrollability (see [Eising, 1984])

$$
\tau(A, B)=\min _{\lambda \in \mathbb{C}} \sigma_{n}([A-\lambda I, \widehat{B}])
$$

(where $\sigma_{n}([A-\lambda I, B])$ denotes the smallest singular value of the augmented matrix $[A-\lambda I, B]$ ) gives a "measure" of how far the original model can be from a non-minimal representation (having thus some entries of the vector $R_{S}^{-1} A(2: n+1,1)$ possibly close to 0$)$. Moreover, it is shown in [Rapaport et al., 2017] how to exploit the transformation to a MRMT structure for minimal representations that are close to non-minimal ones to obtain reduced MRMT models of smaller dimension.

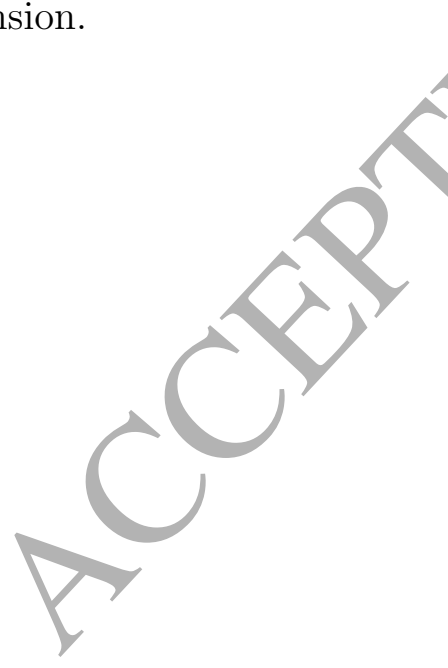




\section{Appendix A2: Conservation of concentrations squared between equivalent SINC mod- els}

We show that the conservation of concentrations squared can be generalized to all SINC models that are equivalent in the sense of Haggerty and Gorelick [1995] (i.e. identity of the mobile concentrations). Consider two equivalent SINC models given by their interaction matrices $A$ and $Z$. Each of these two SINC admits an equivalent MRMT model given by Eq. 14. Being equivalent (and the pairs $(A, B)$ and $(Z, B)$ being controllable), the two MRMT configurations are identical, up to the numbering of the immobile zones. So there exist two transformation matrices $R_{A}$ and $R_{Z}$ from SINC to MRMT such that (Eq. 15):

$$
R_{A} A R_{A}^{-1}=\bar{A}=R_{Z} Z R_{Z}^{-1} .
$$

Eq. 26 implies the conservation of the porosity weighted integral of concentrations squared:

$$
C_{A}^{T} \Phi_{A} C_{A}=\bar{C}^{T} \bar{\Phi} \bar{C}=C_{Z}^{T} \Phi_{Z} C_{Z}
$$

where $C_{A}$ and $\Phi_{A}$ are respectively the concentration vector and the diagonal porosity matrix associated with $A$, and $C_{Z}$ and $\Phi_{Z}$ are their counterparts for $Z$. The relationships between these quantities can be further expressed through the transformation matrix $R_{A Z}$ that transforms the SINC model given by $A$ into the SINC model given by $Z$ :

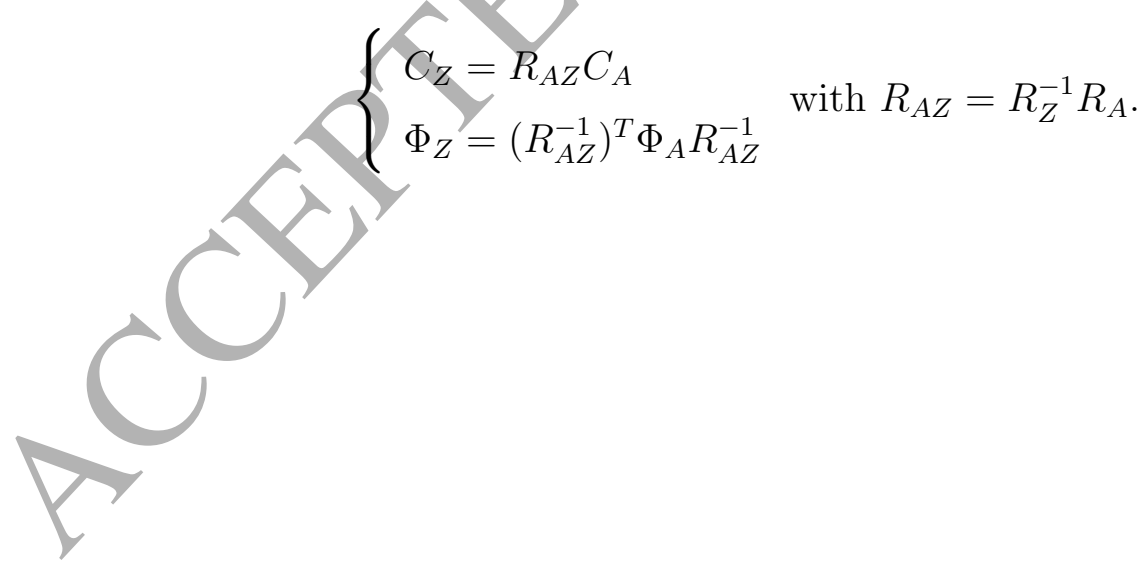




\section{Appendix A3: Conservation of the weighted sum of concentrations squared in planar, cylindrical and spherical inclusions}

The general equation for mobile-immobile models with diffusion into planar, cylindrical or spherical continuous immobile inclusions writes [Haggerty and Gorelick, 1995]:

$$
\frac{\partial c_{m}}{\partial t}+\beta \frac{\partial<c_{i m}>}{\partial t}=L\left(c_{m}\right)
$$

where $c_{m}$ is the mobile concentration, $\left\langle c_{i m}\right\rangle$ is the mean concentration in the immobile domain and $\beta$ is equal to the ratio of the immobile to mobile total porosities. The solution of the PDE in the immobile domain is given by:

$$
c_{i m}(r, t)=\sum_{i=1}^{\infty} a_{i} \frac{f\left(\sqrt{\alpha_{i}} r\right)}{\left\|f\left(\sqrt{\alpha_{i}} r\right)\right\|^{2}} e^{-\alpha_{i} t}
$$

with

$$
a_{i}=\int_{0}^{1} r^{n-1} c_{i m}(r, 0) f\left(\sqrt{\alpha_{i}} r\right) d r
$$

and

$$
\left\|f\left(\sqrt{\alpha_{i}} r\right)\right\|^{2}=\int_{0}^{1} r^{n-1} f^{2}\left(\sqrt{\alpha_{i}} r\right) d r .
$$

For the layered $(n=1)$, cylindrical $(n=2)$ and spherical $(n=3)$ cases, the explicit functions and square norm values for Eq. 44 are given in Table 1, and the values of MRMT rates $\alpha_{i}$ and porosities $\bar{\phi}_{i}$ are given in Table 1 of [Haggerty and Gorelick, 1995].

\begin{tabular}{|c|c|c|c|}
\hline$n$ & 1 & 2 & 3 \\
\hline$f()$. & $\cos ()$. & $I_{0}()$. & $\sin ()$. \\
\hline$\left\|f\left(\sqrt{\alpha_{i}} r\right)\right\|^{2}$ & $\frac{1}{2}$ & $\frac{1}{2} I_{1}^{2}\left(\sqrt{\alpha_{i}} r\right)$ & $\frac{1}{2}$ \\
\hline
\end{tabular}

Table 1: Functions and square norm values of Eq. 44 for $n=1,2,3 . \quad I_{0}$ and $I_{1}$ are the zero-order and first-order modified Bessel functions of the first kind.

By construction, $f$ forms an orthogonal set of functions whatever the dimension of the inclusion. The orthogonality of the function $f$ more generally derives from the theorem of Sturm-Liouville. The Sturm-Liouville theorem states the existence and the orthogonality of the basis function 
for second-order linear differential equations, but does not give their analytical expression. The MRMT model can generally be expressed as:

$$
\bar{c}_{i m, i}(t)=\sum_{i=1}^{\infty} \bar{c}_{i m, i}(t=0) e^{-\alpha_{i} t} .
$$

The relations between the continuous solutions of Eqs. 43-44 and the MRMT model of Eq. 47 provide constrains on the $\bar{\phi}_{i}$ and $\bar{c}_{i m}(t=0)$ :

$$
\bar{\phi}_{i} \bar{c}_{i m}(t=0)=\frac{a_{i}}{\left\|f\left(\sqrt{\alpha_{i}} r\right)\right\|^{2}} \int_{0}^{1} n r^{n-1} f\left(\sqrt{\alpha_{i}} r\right) d r .
$$

Complementary relations derive from the identity of concentrations in the mobile zone:

$$
\int_{0}^{1} c_{i m}(r, t) n r^{n-1} d r=\sum_{i=1}^{\infty} \frac{a_{i}}{\left\|f\left(\sqrt{\alpha_{i}} r\right)\right\|^{2}} \int_{0}^{1} n r^{n-1} f\left(\sqrt{\alpha_{i}} r\right) e^{-\alpha_{i} t} d r .
$$

These equations should be valid whatever the initial conditions and especially for $c_{i m}(r, t=0)=1$ which corresponds to $\bar{c}_{i m, i}(t=0)=1$ for all immobile zoness $i$, like in Eq. 19 and in the Appendix B of [Haggerty and Gorelick, 1995]. In such a case the MRMT porosities $\bar{\phi}_{i}$ can be straightforwardly identified as:

$$
\begin{aligned}
\bar{\phi}_{i} & =\frac{1}{n\left\|f\left(\sqrt{\alpha_{i}} r\right)\right\|^{2}}\left(\int_{0}^{1} n r^{n-1} f\left(\sqrt{\alpha_{i}} r\right) d r\right)^{2} \\
& =\frac{1}{n} \frac{\left(\int_{0}^{1} n r^{n-1} f\left(\sqrt{\alpha_{i}} r\right) d r\right)^{2}}{\int_{0}^{1} r^{n-1} f^{2}\left(\sqrt{\alpha_{i}} r\right) d r}
\end{aligned}
$$

The initial MRMT concentrations are given by:

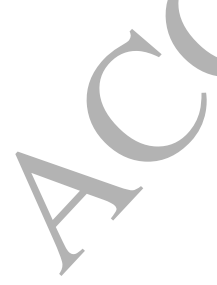

$$
\bar{c}_{i m, i}(t=0)=\frac{\int_{0}^{1} n r^{n-1} f\left(\sqrt{\alpha_{i}} r\right) c_{i m}(r, t=0) d r}{\int_{0}^{1} n r^{n-1} f\left(\sqrt{\alpha_{i}} r\right) d r} .
$$

$\bar{c}_{i m, i}(t=0)$ is simply some normalized scalar product of the initial condition $c_{i m, i}(t=0)$ by the basis function corresponding to $\alpha_{i}$. The sum of the concentrations squared weighted by the 
porosities $\Sigma$ for the continuous formulation of the solution writes:

$$
\begin{aligned}
\Sigma & =n \int_{0}^{1} r^{n-1} c_{i m}^{2}(r, t) d r \\
& =n \sum_{i=1}^{\infty} \frac{a_{i}^{2}}{\left\|f\left(\sqrt{\alpha_{i}} r\right)\right\|^{4}} e^{-2 \alpha_{i} t} \int_{0}^{1} r^{n-1} f^{2}\left(\sqrt{\alpha_{i}} r\right) d r \\
& =n \sum_{i=1}^{\infty} \frac{a_{i}^{2}}{\left\|f\left(\sqrt{\alpha_{i}} r\right)\right\|^{2}} e^{-2 \alpha_{i} t} .
\end{aligned}
$$

The sum of the concentrations squared weighted by the porosities $\bar{\Sigma}$ in the equivalent MRMT 327 writes:

$$
\begin{aligned}
\bar{\Sigma} & =\sum_{i=1}^{\infty} \bar{\phi}_{i} \bar{c}_{i m, i}^{2}(t=0) e^{-2 \alpha_{i} t} \\
& =\sum_{i=1}^{\infty} \frac{\left(\int_{0}^{1} n r^{n-1} f\left(\sqrt{\alpha_{i}} r\right) d r\right)^{2}}{n \int_{0}^{1} r^{n-1} f^{2}\left(\sqrt{\alpha_{i}} r\right) d r} \frac{n^{2} a_{i}^{2}}{\left(\int_{0}^{1} n r^{n-1} f\left(\sqrt{\alpha_{i}} r\right) d r\right)^{2}} e^{-2 \alpha_{i} t} \\
& =n \sum_{i=1}^{\infty} \frac{a_{i}^{2}}{\left\|f\left(\sqrt{\alpha_{i}} r\right)\right\|^{2}} e^{-2 \alpha_{i} t} \\
& =\Sigma .
\end{aligned}
$$




\section{References}

\section{References}

Andréa-Novel, B. and de Lara, M. (2013). Control Theory for Engineers: A Primer. Springer, Berlin.

Babey, T., de Dreuzy, J.-R., and Casenave, C. (2015). Multi-rate mass transfer (MRMT) models for general diffusive porosity structures. Advances in Water Resources, 76:146-156.

Babey, T., de Dreuzy, J. R., and Ginn, T. R. (2016). From conservative to reactive transport under diffusion-controlled conditions. Water Resources Research, 52(5):3685-3700.

Beaudoin, A., Huberson, J.-R., and de Dreuzy, J.-R. (2017). Adapting particle methods to model the dynamics of concentration gradients and chemical reactivity under advective diffusive transport conditions. Journal of Computational Physics. Accepted for publication.

Benson, D. A., Wheatcraft, S. W., and Meerschaert, M. M. (2000). Application of a fractional advection-dispersion equation. Water Resources Research, 36(6):1403-1412.

Berkowitz, B., Cortis, A., Dentz, M., and Scher, H. (2006). Modeling non-fickian transport in geological formations as a continuous time random walk. Reviews of Geophysics, 44(2):RG2003.

Bijeljic, B., Mostaghimi, P., and Blunt, M. J. (2013). Insights into non- fickian solute transport in carbonates. Water Resources Research, 49(5):2714-2728.

Bouchaud, J.-P. and Georges, A. (1990). Anomalous diffusion in disordered media: Statistical mechanisms, models and physical applications. Physics Reports, 195(4-5):127-293.

Briggs, M. A., Day-Lewis, F. D., Zarnetske, J. P., and Harvey, J. W. (2015). A physical explanation for the development of redox microzones in hyporheic flow. Geophysical Research Letters, $42(11): 4402-4410$.

Carrera, J., Snchez-Vila, X., Benet, I., Medina, A., Galarza, G., and Guimerá, J. (1998). On matrix diffusion: formulations, solution methods and qualitative effects. Hydrogeology Journal, 6(1):178-190.

Chen, C.-T. (1999). Linear System Theory and Design. Oxford University Press, Inc., New York, NY, USA, 3rd edition. 
Davy, P., Le Goc, R., Darcel, C., Bour, O., de Dreuzy, J. R., and Munier, R. (2010). A likely universal model of fracture scaling and its consequence for crustal hydromechanics. Journal of Geophysical Research-Solid Earth, 115:13.

de Dieuleveult, C., Erhel, J., and Kern, M. (2009). A global strategy for solving reactive transport equations. Journal of Computational Physics, 228(17):6395 - 6410.

de Dreuzy, J. R. and Carrera, J. (2016). On the validity of effective formulations for transport through heterogeneous porous media. Hydrol. Earth Syst. Sci., 20(4):1319-1330.

de Dreuzy, J.-R., Carrera, J., Dentz, M., and Le Borgne, T. (2012). Time evolution of mixing in heterogeneous porous media. Water Resources Research, 48(6). W06511

de Dreuzy, J.-R., Rapaport, A., Babey, T., and Harmand, J. (2013). Influence of porosity structures on mixing-induced reactivity at chemical equilibrium in mobile/immobile multi-rate mass transfer (MRMT) and multiple interacting continua (MINC) models. Water Resources Research, 49(12):8511-8530.

De Simoni, M., Carrera, J., Snchez-Vila, X., and Guadagnini, A. (2005). A procedure for the solution of multicomponent reactive transport problems. Water Resources Research, 41(11). W11410.

De Simoni, M., Sanchez-Vila, X., Carrera, J., and Saaltink, M. W. (2007). A mixing ratios-based formulation for multicomponent reactive transport. Water Resources Research, 43(7). W07419.

Delhomme, J. P. (1979). Spatial variability and uncertainty in groundwater flow parameters: A geostatistical approach. Water Resources Research, 15(2):269-280.

Dentz, M. and Berkowitz, B. (2003). Transport behavior of a passive solute in continuous time random walks and multirate mass transfer. Water Resources Research, 39(5). 1111.

Donado, L., Sanchez-Vila, X., Dentz, M., Carrera, J., and Bolster, D. (2009). Multicomponent reactive transport in multicontinuum media. Water Resources Research, 45(11). W11402.

Eising, R. (1984). Between controllable and uncontrollable. System \& Control Letters, 4(5):263265. 
Fernandez-Garcia, D., Llerar-Meza, G., and Gomez-Hernandez, J. J. (2009). Upscaling transport with mass transfer models: Mean behavior and propagation of uncertainty. Water Resources Research, 45(10). W10411.

Geiger, S., Dentz, M., and Neuweiler, I. (2013). A novel multi-rate dual-porosity model for improved simulation of fractured and multiporosity reservoirs. SPE-148130-PA.

Gelhar, L. W. and Axness, C. L. (1983). Three-dimensional stochastic analysis of macrodispersion in aquifers. Water Resources Research, 19:161-180.

Gjetvaj, F., Russian, A., Gouze, P., and Dentz, M. (2015). Dual control of flow field heterogeneity and immobile porosity on non-fickian transport in berea sandstone. Water Resources Research, 51(10):8273-8293.

Godsil, C. and Royle, G. (2001). Algebraic Graph Theory. Graduate Text in Mathematics. Springer.

Gouze, P., Melean, Y., Le Borgne, T., Dentz, M., and Carrera, J. (2008). Non-fickian dispersion in porous media explained by heterogeneous microscale matrix diffusion. Water Resources Research, 44(11). W11416.

Gramling, C. M., Harvey, C. F., and Meigs, L. C. (2002). Reactive transport in porous media: A comparison of model prediction with laboratory visualization. Environmental Science and Technology, 36(11):2508-2514.

Greskowiak, J., Hay, M. B., Prommer, H., Liu, C. X., Post, V. E. A., Ma, R., Davis, J. A., Zheng, C. M., and Zachara, J. M. (2011). Simulating adsorption of U(VI) under transient groundwater flow and hydrochemistry: Physical versus chemical nonequilibrium model. Water Resources Research, 47(8). W08501.

Haggerty, R. and/Gorelick, S. (1995). Multiple-rate mass transfer for modeling diffusion and surface reactions in media with pore-scale heterogeneity. Water Resources Research, 31(10):2383-2400.

Haggerty, R., Harvey, C. F., von Schwerin, C. F., and Meigs, L. C. (2004). What controls the apparent timescale of solute mass transfer in aquifers and soils? A comparison of experimental results. Water Resources Research, 40(1). W01510.

Havlin, S. and Ben-Avraham, D. (1987). Diffusion in disordered media. Advances in Physics, $36(6): 695-798$ 
Henri, C. V. and Fernandez-Garcia, D. (2015). A random walk solution for modeling solute transport with network reactions and multi-rate mass transfer in heterogeneous systems: Impact of biofilms. Advances in Water Resources, 86, Part A:119-132.

Jimenez-Martinez, J., de Anna, P., Tabuteau, H., Turuban, R., Le Borgne, T., and Meheust, Y. (2015). Pore-scale mechanisms for the enhancement of mixing in unsaturated porous media and implications for chemical reactions. Geophysical Research Letters, 42(13):5316-5324.

Kailath, T. (1980). Linear Systems. Prentice Hall.

Karimi-Fard, M. and Durlofsky, L. (2016). A general gridding, discretization, and coarsening methodology for modeling flow in porous formations with discrete geological features. Advances in Water Resources, 96:354-372.

Karimi-Fard, M., Gong, B., and Durlofsky, L. J. (2006). Generation of coarse-scale continuum flow models from detailed fracture characterizations. Water Resources Research, 42(10). W10423.

Knorr, B., Maloszewski, P., Krmer, F., and Stumpp, C. (2016). Diffusive mass exchange of non-reactive substances in dual-porosity porous systems column experiments under saturated conditions. Hydrological Processes, 30(6):914 926. HYP-15-0258.R1.

Le Borgne, T., Dentz, M., Bolster, D., Carrera, J., de Dreuzy, J.-R., and Davy, P. (2010). Nonfickian mixing: Temporal evolution of the scalar dissipation rate in heterogeneous porous media. Advances in Water Resources, 3(12):1468-1475.

Le Borgne, T., Dentz, M., Davy, P., Bolster, D., Carrera, J., de Dreuzy, J.-R., and Bour, O. (2011). Persistence of incomplete mixing: A key to anomalous transport. Phys. Rev. E, 84:015301.

Le Borgne, T., Dentz,M., and Villermaux, E. (2015). The lamellar description of mixing in porous media. Journal of Fluid Mechanics, 770:458498.

Le Borgne, T. and Gouze, P. (2008). Non-fickian dispersion in porous media: 2. model validation from measurements at different scales. Water Resources Research, 44(6). W06427.

Li, L., Zhou, H., and Gómez-Hernández, J. (2011). Transport upscaling using multi-rate mass transfer in three-dimensional highly heterogeneous porous media. Advances in Water Resources, $34(4): 478-489$ 
Lichtner, P. C. and Kang, Q. (2007). Upscaling pore-scale reactive transport equations using a multiscale continuum formulation. Water Resources Research, 43(12):19. W12S15.

Luquot, L., Roetting, T. S., and Carrera, J. (2014). Characterization of flow parameters and evidence of pore clogging during limestone dissolution experiments. Water Resources Research, 50(8):6305-6321.

Neuman, S. P. and Tartakovsky, D. M. (2009). Perspective on theories of non-fickian transport in heterogeneous media. Advances in Water Resources, 32(5):670-680.

Noetinger, B., Roubinet, D., Russian, A., Le Borgne, T., Delay, F., Dentz, M., de Dreuzy, J.-R., and Gouze, P. (2016). Random walk methods for modeling hydrodynamic transport in porous and fractured media from pore to reservoir scale. Transport in Porous Media, 115(2):345-385.

Park, Y.-J., Lee, K.-K., Kosakowski, G., and Berkowitz, B. (2003). Transport behavior in threedimensional fracture intersections. Water Resources Research, 39(1). 1215.

Pool, M., Post, V., and Simmons, C. (2015). Effects of tidal fluctuations and spatial heterogeneity on mixing and spreading in spatially heterogeneous coastal aquifers. Water Resources Research, 51(3):1570-1585.

Pruess, K. and Narasimhan, T. N. (1985). A practical method for modeling fluid and heat-flow in fractured porous-media. Society of Petroleum Engineers Journal, 25(1):14-26.

Rapaport, A., Rojas-Palma, A., de Dreuzy, J.-R., and Ramirez, H. (2017). Equivalence of finite dimensional input-output models of solute transport and diffusion in geosciences. IEEE Transactions on Automatic Control, 62(10). on line.

Roubinet, D., de Dreuzy, J.-R., and Tartakovsky, D. M. (2013). Particle-tracking simulations of anomalous transport in hierarchically fractured rocks. Computers and Geosciences, 50(0):52-58.

Rubin, J. (1983). Transport of reacting solutes in porous media: Relation between mathematical nature of problem formulation and chemical nature of reactions. Water Resources Research, 19(5):1231-1252.

Sanchez-Vila, X., Fernandez-Garcia, D., and Guadagnini, A. (2010). Interpretation of column experiments of transport of solutes undergoing an irreversible bimolecular reaction using a continuum approximation. Water Resources Research, 46(12). W12510. 
Scheibe, T. D., Hou, Z. S., Palmer, B. J., and Tartakovsky, A. M. (2013). Pore-scale simulation of intragranular diffusion: Effects of incomplete mixing on macroscopic manifestations. Water Resources Research, 49(7):4277-4294.

Silva, O., Carrera, J., Dentz, M., Kumar, S., Alcolea, A., and Willmann, M. (2009). A general realtime formulation for multi-rate mass transfer problems. Hydrology and Earth System Sciences, 13(8):1399-1411.

Soler-Sagarra, J., Luquot, L., Martinez-Perez, L., Saaltink, M. W., De Gaspari, F., and Carrera, J. (2016). Simulation of chemical reaction localization using a multi-porosity reactive transport approach. International Journal of Greenhouse Gas Control, 48:59-68.

Steefel, C., DePaolo, D., and Lichtner, P. (2005). Reactive transport modeling: An essential tool and a new research approach for the earth sciences. Earth and Planetary Science Letters, $240(3): 539-558$.

Tyukhova, A., Kinzelbach, W., and Willmann, M. (2015). Delineation of connectivity structures in 2-d heterogeneous hydraulic conductivity fields. Water Resources Research, 51(7):5846-5854.

Tyukhova, A. and Willmann, M. (2016). Conservative transport upscaling based on information of connectivity. Water Resources Research, 52(9):6867-6880.

Willmann, M., Carrera, J., and Sanchez-Vila, X. (2008). Transport upscaling in heterogeneous aquifers: What physical parameters control memory functions? Water Resources Research, 44(12). W12437.

Willmann, M., Carrera, J., Sanchez-Vila, X., Silva, O., and Dentz, M. (2010). Coupling of mass transfer and reactive transport for nonlinear reactions in heterogeneous media. Water Resources Research, 46(7). W07512.

Zhang, Y., Green, C., and Baeumer, B. (2014). Linking aquifer spatial properties and non-fickian transport in mobileimmobile like alluvial settings. Journal of Hydrology, 512:315 - 331.

Zhang, Y., Green, C., and Fogg, G. (2013). The impact of medium architecture of alluvial settings on non-fickian transport. Advances in Water Resources, 54:78 - 99.

Zinn, B., Meigs, L. C., Harvey, C. F., Haggerty, R., Peplinski, W. J., and Von Schwerin, C. F. (2004). Experimental visualization of solute transport and mass transfer processes in two- 
493 dimensional conductivity fields with connected regions of high conductivity. Environmental $494 \quad$ Science and Technology, 38(14):3916-3926.

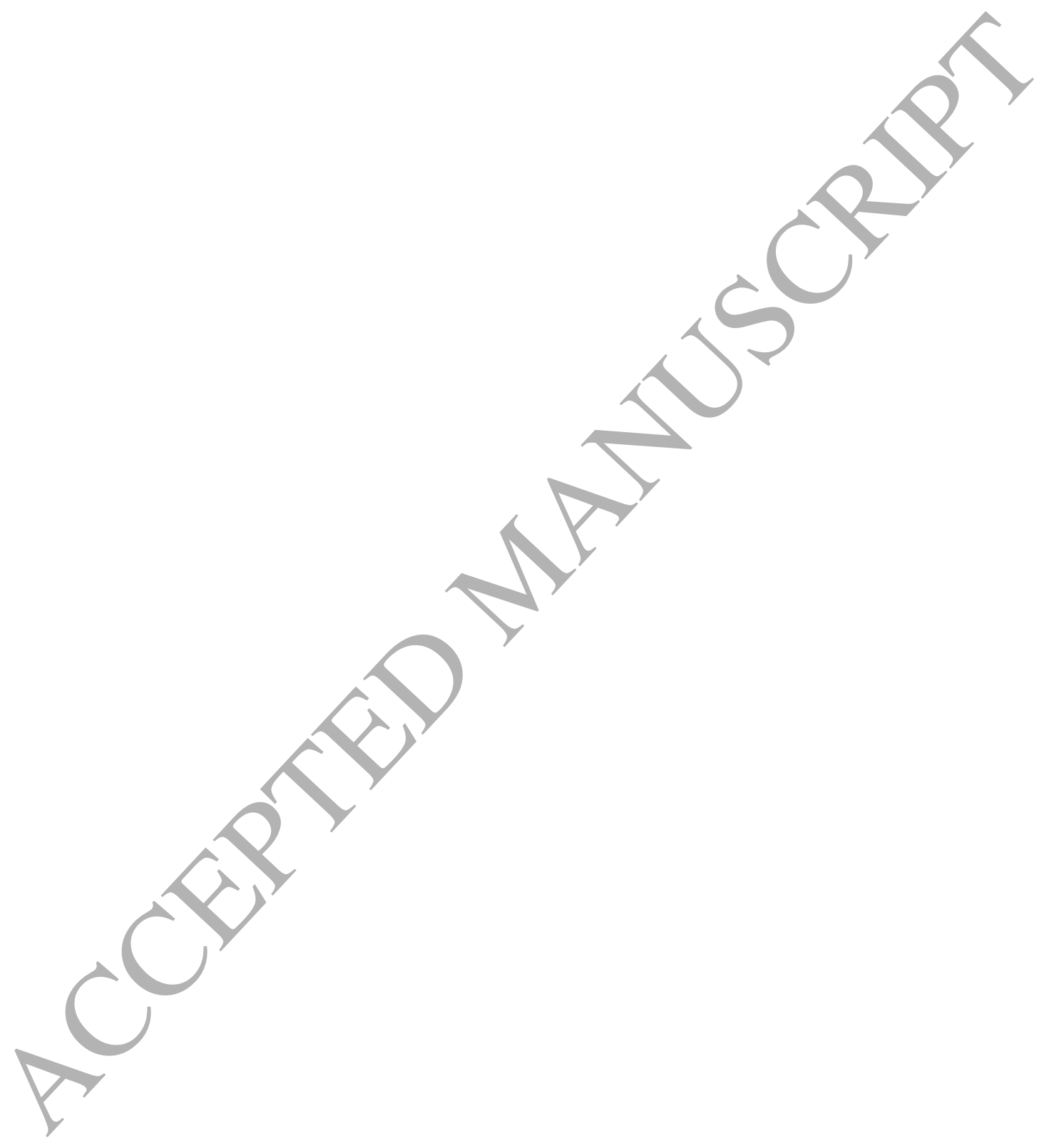

